\title{
The Pub1 and Upf1 Proteins Act in Concert to Protect Yeast from Toxicity of the $\left[\mathrm{PSI}^{+}\right]$Prion
}

\author{
Valery N. Urakov, Olga V. Mitkevich *, Alexander A. Dergalev and Michael D. Ter-Avanesyan ${ }^{\dagger}$ \\ Bach Institute of Biochemistry, Federal Research Center "Fundamentals of Biotechnology" of the Russian \\ Academy of Sciences, 119071 Moscow, Russia; valery.urakov@gmail.com (V.N.U.); \\ alexanderdergalioff@gmail.com (A.A.D.) \\ * Correspondence: mitkevich@inbi.ras.ru; Tel.: +7-495-954-40-97 \\ † Michael D. Ter-Avanesyan passed away on 25 October 2018.
}

Received: 8 October 2018; Accepted: 14 November 2018; Published: 20 November 2018

\begin{abstract}
The $\left[\mathrm{PSI}^{+}\right]$nonsense-suppressor determinant of Saccharomyces cerevisiae is based on the formation of heritable amyloids of the Sup35 (eRF3) translation termination factor. [PSI ${ }^{+}$amyloids have variants differing in amyloid structure and in the strength of the suppressor phenotype. The appearance of $\left[\mathrm{PSI}^{+}\right]$, its propagation and manifestation depend primarily on chaperones. Besides chaperones, the Upf1/2/3, Siw14 and Arg82 proteins restrict $\left[\mathrm{PSI}^{+}\right]$formation, while Sla2 can prevent $\left[\mathrm{PSI}^{+}\right]$toxicity. Here, we identify two more non-chaperone proteins involved in $\left[\mathrm{PSI}^{+}\right]$ detoxification. We show that simultaneous lack of the Pub1 and Upf1 proteins is lethal to cells harboring $\left[\mathrm{PSI}^{+}\right]$variants with a strong, but not with a weak, suppressor phenotype. This lethality is caused by excessive depletion of the Sup45 (eRF1) termination factor due to its sequestration into Sup35 polymers. We also show that Pub1 acts to restrict excessive Sup35 prion polymerization, while Upf1 interferes with Sup45 binding to Sup35 polymers. These data allow consideration of the Pub1 and Upf1 proteins as a novel $\left[\mathrm{PSI}^{+}\right]$detoxification system.
\end{abstract}

Keywords: Saccharomyces cerevisiae; $\left[\mathrm{PSI}^{+}\right]$prion toxicity; translation termination factors; Sup35; Sup45; Pub1; Upf1

\section{Introduction}

Similar to other amyloids, most prions are formed in a process of highly ordered non-covalent polymerization of partially misfolded protein monomers. The ability to form amyloids is a common inherent feature of conformationally flexible proteins, which in many cases contain intrinsically disordered domains and, since such proteins are widespread in nature, amyloids are found in a wide range of organisms from mammals to bacteria, where they can have both deleterious and beneficial effects $[1,2]$. While in mammals, prions cause neurodegenerative diseases, in fungi they mediate non-chromosomal inheritance of several phenotypic traits [3,4]. Importantly, due to the high genetic tractability of Saccharomyces cerevisiae, its prions, and especially $\left[\mathrm{PSI}^{+}\right]$, are the most well studied. $\left[\mathrm{PSI}^{+}\right]$is a prion determinant that gives rise to a nonsense suppressor phenotype as a consequence of the amyloid aggregation and partial inactivation of the translation termination factor Sup35 (eRF3) [5-7]. Prionization of Sup35 can result in appearance of multiple [PSI ${ }^{+}$] variants that differ by the strength of their nonsense suppressor phenotype and stability of inheritance $[8,9]$. The dissimilarity in the properties of $\left[\mathrm{PSI}^{+}\right]$variants reflects heritable differences in the structure of Sup35 prion polymers [10,11]. Although the process of prion polymerization is autocatalytic, in vivo the appearance of $\left[\mathrm{PSI}^{+}\right]$, as well as its propagation and manifestation depend on the activity of chaperones (for a review, see Reference [12]). Besides chaperones, some non-chaperone proteins interacting with prion-forming proteins can also influence the properties of prion amyloids. 
For example, Sla1-mediated interaction of Sup35 with the actin cytoskeleton was shown to promote generation of the $\left[\mathrm{PSI}^{+}\right]$prion [13]. The interaction of Sup35 with the Sup45 (eRF1) termination factor has two effects, it decreases prion formation [14] and it can contribute to $\left[\mathrm{PSI}^{+}\right]$toxicity $[15,16]$.

The biological significance of yeast prions, and the $\left[\mathrm{PSI}^{+}\right]$prion in particular, is still subject to debate [17-22]. Here, we confirm the role of Pub1 in Sup35 polymerization [23] and also show that the Upf1 protein, involved in the control of the nonsense codon-mediated mRNA decay (NMD) [24], decreases sequestration of Sup45 into Sup35 prion polymers. Individually, the Pub1 and Upf1 proteins do not alleviate the harmful effects of $\left[\mathrm{PSI}^{+}\right]$, but their combined action can suppress $\left[\mathrm{PSI}^{+}\right]$toxicity and, therefore, these proteins can be viewed as a $\left[\mathrm{PSI}^{+}\right]$detoxification system.

\section{Results}

\subsection{Simultaneous Deletion of PUB1 and UPF1 in the Presence of $\left[\mathrm{PSI}^{+}\right]$Can Be Synthetic Lethal}

The work was inspired by an incidental observation made during elucidation of the role of Pub1 in translation termination [23], demonstrating that the UPF1 gene could be deleted in the 74-D694 strain with deleted $P U B 1$, only if this strain did not carry strong $\left[P S I^{+}\right]_{S 7}$. This indicated that simultaneous deletion of PUB1 (pub1- $\Delta)$ and UPF1 (upf1- $\Delta)$ in the presence of $\left[P^{+} I^{+}\right]$caused synthetic lethality. To confirm this, we deleted UPF1 in the transformants of 74-D694 $\left[\mathrm{PSI}^{+}\right]_{\mathrm{S7}}$ deleted for PUB1, which carry the wild-type PUB1 gene on multicopy plasmids with either LEU2 or URA3. In the obtained strains, these plasmids could not be changed for the empty vectors with complementary markers, though they were easily interchangeable for the multicopy PUB1 or UPF1 plasmids with appropriate selectable markers. (Table 1). These experiments showed that the 74-D694 strain with PUB1 and UPF1 deletions could grow only if it did not contain $\left[\mathrm{PSI}^{+}\right]_{\mathrm{S} 7}$ or expressed plasmid-encoded copies of the PUB1 or UPF1 genes.

Table 1. Overproduction of Upf1, Pub1, Pub1 $\triangle$ C, Sup45 and Sup35C rescues the $\left[P S I^{+}\right]_{S 7}$ pub1- $\Delta$ upf1- $\Delta$ cells from lethality.

\begin{tabular}{ccc}
\hline Plasmid & Rescue Plasmid Loss (\%) & Suppression of Synthetic Lethality \\
\hline Multi-UPF1 & 36 & + \\
CEN-UPF1 & 52 & + \\
${ }^{*}$ Multi-PUB1 & 86 & + \\
${ }^{*}$ CEN-PUB1 & 87 & + \\
${ }^{*}$ Multi-pub1- $\triangle C$ & 36 & + \\
${ }^{*}$ CEN- pub1- $\Delta C$ & 50 & + \\
Multi-SUP45 & 84 & + \\
CEN-SUP45 & 53 & + \\
Multi-sup35-C & 38 & - \\
CEN- sup35-C & 0 & - \\
Empty vector & 0 & + \\
\hline
\end{tabular}

Multi and CEN, multicopy and centromeric plasmids, respectively. Transformants carried two plasmids, the rescue plasmid with wild-type PUB1 and with either LEU2 or URA3 as selectable markers (YEplac181-PUB1 or YEplac195-PUB1, respectively) and the other with the tested gene and appropriate selectable marker. As a control, the empty vectors YEplac195 or YEplac181 with either URA3 or LEU2, respectively, were used. Transformants were streaked on SC medium selective for the marker of plasmid carrying the tested gene. For each transformant, more than 100 clones growing up were examined. The percentage of clones that lost the rescue plasmid, was calculated. + and - indicate ability or inability of the plasmid to suppress synthetic lethality, respectively, which is concluded from the ability to lose the rescue plasmid. * Transformant, the growth rate of which was studied (Figure 1b).

In contrast to UPF1, the molecular mechanism responsible for the PUB1 rescuing effect is clear, since adding a PUB1 wild-type allele prevents the increase of Sup35 polymerization caused by pub1- $\Delta$, which is most probably due to the ability of Pub1 to interact with Sup35 [23]. However, to our surprise, the plasmids encoding the Pub1 variant without a C-terminal extension (Pub1 $\Delta \mathrm{C}$ ) which contains the major site through which Pub1 interacts with Sup35, also suppressed synthetic lethality. This suggested that weak interaction with Sup35 mediated by the Pub1 internal low-affinity 
binding site [23] was sufficient for inhibition of Sup35 polymerization. To test this suggestion, we compared the amount of Sup35 polymers in the 74-D694 $\left[P S I^{+}\right]_{S 7}$ strain containing chromosomal pub1- $\Delta$ and plasmids with either pub1- $\Delta C$ or wild-type PUB1, or an empty vector. In accordance with our suggestion, pub1- $\Delta C$ caused a small, but statistically significant decrease in the amount of Sup35 polymers (Figure 1a). Importantly, suppression of synthetic lethality by pub1- $\Delta C$ was also incomplete, as revealed by a decreased growth rate of transformants carrying the pub1- $\Delta C$ plasmid compared to growth of transformants with the plasmid bearing wild-type PUB1 (Figure 1b). It is also notable that although we showed earlier that pub1- $\Delta$ causes an approximately 2 -fold increase in the amount of Sup35 polymers in the cells with $\left[\mathrm{PSI}^{+}\right]_{S 7}[23]$, in this work this difference was only about 1.5-fold. This discrepancy could be due to different growth conditions. Indeed, in an earlier work we compared strains with the chromosomal pub1- $\triangle$ and PUB1 alleles, grown in rich YPD medium (see Materials and Methods), whereas here we examined levels of Sup35 polymers in transformants grown in synthetic medium selective for the plasmid marker.

Next, we examined the ability of the Sup35C protein lacking the $\mathrm{N}$-terminal prion-forming domain to suppress synthetic lethal interaction between pub1- $\Delta$, upf1- $\Delta$ and $\left[P S I^{+}\right]_{S 7}$. It is known that due to the absence of the prion domain, Sup35C cannot polymerize in $\left[\mathrm{PSI}^{+}\right]$cells, though it retains the ability to bind Sup45, thus interfering with sequestration of Sup45 into Sup35 polymers [5,25]. However, only multicopy sup35-C plasmid ensured cell viability, thus suggesting that high levels of soluble Sup35C were required for sufficient binding of Sup45, which in turn prevents its sequestration. Finally, the role of Sup45 depletion in synthetic lethality was proved by the ability of its overproduction to rescue lethality of the pub1- $\triangle$ upf1- $\triangle\left[P S I^{+}\right]_{S 7}$ strain (Table 1).

It should be noted that UPF1 controls NMD in concert with the UPF2 and UPF3 genes and deletion of any one of them completely abolishes decay of nonsense-containing mRNAs [26]. Importantly, besides NMD, these genes also control nonsense codon readthrough, and deletion of each of them increases readthrough to approximately the same level [27]. However, despite this functional similarity, deletion of either UPF2 or UPF3, as well as simultaneous deletion of these genes in the 74-D694 $\left[\mathrm{PSI}^{+}\right]_{\mathrm{S}}$ strain deleted for PUB1 did not cause cell lethality, as was shown by the ability of these deletants to lose the rescue LEU2 YEplac181-PUB1 plasmid: streaking cells of corresponding transformants on YPD plates gave rise to $41 \%, 54 \%$ and $38 \%$ Leu $^{-}$clones, respectively (approximately 200 clones were tested for each transformant). This indicates that the observed synthetic lethality was not the consequence of an NMD defect or the increase of nonsense codon readthrough caused by the UPF1 deletion.

The type of $\left[\mathrm{PSI}^{+}\right]$was important for the synthetic lethality. Simultaneous deletion of PUB1 and UPF1 in the same yeast strain carrying weak $\left[\mathrm{PSI}^{+}\right]$variants, $\left[\mathrm{PSI}^{+}\right]_{\mathrm{WS} 2}$ and $\left[P S I^{+}\right]_{\mathrm{W} 2}$ (Table 2 ), was not lethal, and corresponding transformants could easily lose the PUB1 LEU2 rescue plasmid. Among approximately 200 clones growing in nonselective YPD medium, 22\% and 36\% were Leu', respectively. Thus, the ability to cause synthetic lethality correlated with the strength of the $\left[\mathrm{PSI}^{+}\right]$ suppressor phenotype.

Table 2. Efficiency of nonsense codon readthrough caused by different $\left[\mathrm{PSI}^{+}\right]$variants.

\begin{tabular}{cc}
\hline$\left[\mathrm{PSI}^{+}\right]$Variant & \% Readthrough \\
\hline$\left[\mathrm{PSI}^{+}\right]_{\mathrm{S} 7}$ & $6.1 \pm 0.4$ \\
{$\left[P S I^{+}\right]_{\mathrm{WS} 2}$} & $2.1 \pm 0.1$ \\
{$\left[\mathrm{PSI}^{+}\right]_{\mathrm{W} 2}$} & $1.5 \pm 0.2$ \\
\hline
\end{tabular}

The UGAC stop signal was used for measurement, which shows the highest readthrough among all stop codons $[23,28]$. Percent readthrough is expressed as the mean \pm SEM. 
(a)
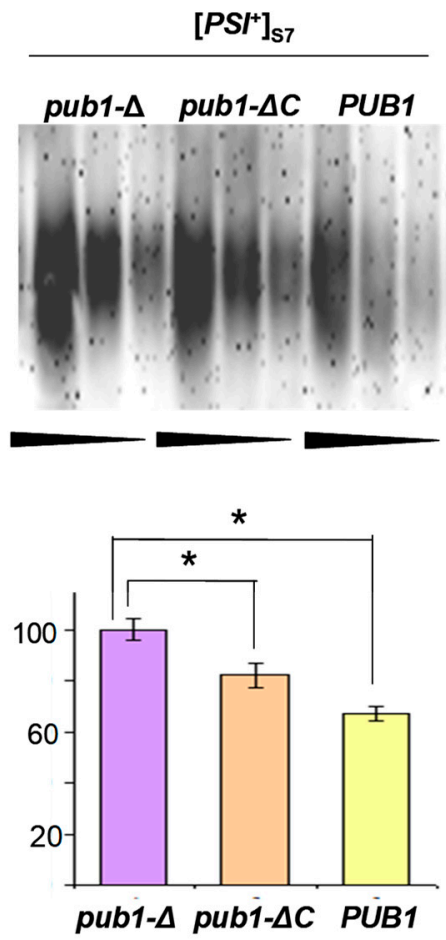

(b)

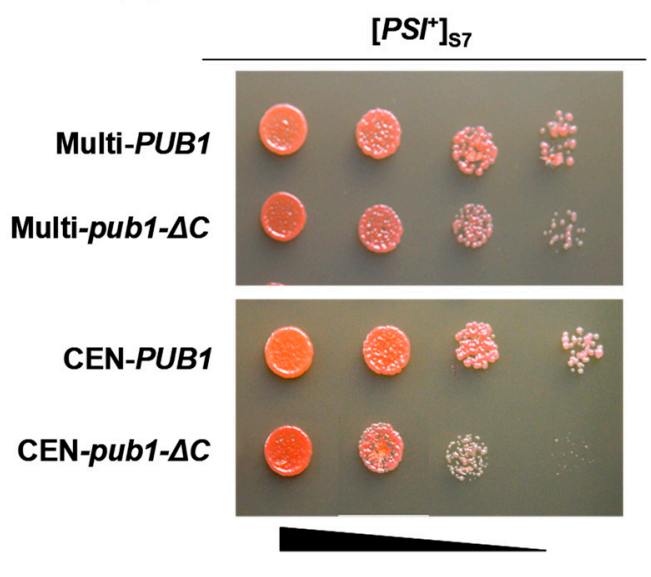

Figure 1. Plasmid-encoded Pub1 $\triangle \mathrm{C}$ slightly compensates the effect of chromosomal pub1- $\Delta$ on prion polymerization of Sup 35 in $\left[\mathrm{PSI}^{+}\right]_{57}$ cells and alleviates the synthetic lethal interaction between $p u b 1-\Delta$, upf1- $\triangle$ and $\left[\mathrm{PSI}^{+}\right]_{\mathrm{S7}}$. (a) SDD-AGE analysis of polymerized Sup35 in transformants of the 74-D694 $\left[\mathrm{PSI}^{+}\right]_{\mathrm{S} 7}$ strain, with PUB1 deletion carrying multicopy plasmids encoding wild-type Pub1 (PUB1), Pub1 $\Delta C$ (pub1- $\Delta C)$, or empty vector (pub1- $\Delta$ ). The transformants were grown in liquid Sc-Ura medium selective for the plasmid marker. Blots were probed with the polyclonal antibody against Sup35NM. Equal amounts of total protein from the compared cell lysates were serially diluted in two-fold decrements. Undiluted samples contained $\sim 180 \mu \mathrm{g}$ of total protein per lane. Four independent transformants of each type were analyzed and representative blot images are presented. Abundances of polymerized Sup35 (percent of the level in the pub1- $\Delta$ strain \pm SEM) were calculated after densitometry of blots and are shown on the histograms. Statistically significant differences of polymerized Sup35 in compared transformants (marked by trapezoid), determined by Student's $t$-test, are indicated by asterisks $\left.{ }^{*} p<0.05\right)$. (b) Growth of the transformants on the solid SC-Ura medium. The transformants of the pub1- $\triangle$ upf1- $\triangle\left[P S I^{+}\right]_{S 7}$ strain with multicopy YEplac195-PUB1 (Multi-PUB1), YEplac195-PUB1 $\triangle C$ (Multi-pub1- $\Delta C$ ) and centromeric pRS316-PUB1 (CEN-PUB1), pRS316-PUB1 $\triangle C$ C (CEN-pub1- $\Delta C$ ) plasmids were grown in liquid SC-Ura medium and after $12 \mathrm{~h}$ incubation, cell suspensions were diluted to an $\mathrm{OD}_{600}$ of 0.3 , spotted onto plates with the same medium and incubated for four days at $30^{\circ} \mathrm{C}$. Four serial three-fold dilutions of cell suspensions are shown.

\subsection{The Lack of Pub1 But Not of Upf1 Increases Prion Polymerization of Sup35}

The ability of overproduced Sup45 to suppress synthetic lethality of PUB1 and UPF1 deletions in the $\left[\mathrm{PSI}^{+}\right]_{\mathrm{S}}$ background suggested that this lethality resulted from a depletion of soluble Sup45 caused by its sequestration into Sup 35 prion polymers. The deficiency of Sup 45 could be aggravated by inhibition of SUP45 expression by UPF1 or PUB1 deletion. However, the levels of Sup45 and Sup35 in the [psi] strain were not affected by either pub1- $\Delta$ [23], or upf1- $\Delta$ (Figure S1). Since pub1- $\Delta$ significantly increases the level of Sup35 polymers, it was reasonable to suggest that the deletion of UPF1 also causes an increase of Sup35 polymerization, and together these deletions increase Sup35 polymerization to the level which is incompatible with cell viability. However, comparison of the amount of Sup35 polymers in the 74-D694 $\left[\mathrm{PSI}^{+}\right]_{S 7}$ strain carrying either wild-type or deleted UPF1 did not reveal any effects of this gene on Sup35 polymerization. Importantly, deletions of UPF2 or UPF3 genes also did not influence the level of Sup35 prion polymers in this strain (Figure S2). 
The role of Pub1 in Sup35 prion polymerization was shown only for one [PSI $\left.{ }^{+}\right]_{S 7}$ variant [23]. To elucidate whether the effect of $p u b 1-\Delta$ on Sup35 polymerization was $\left[\mathrm{PSI}^{+}\right]$variant-specific or not, we tested it in the same strain which carried $\left[\mathrm{PSI}^{+}\right]$variants with a weak suppressor phenotype. Analysis of Sup35 polymerization in the strains with $\left[\mathrm{PSI}^{+}\right]_{\mathrm{W} 2}$ and $\left[\mathrm{PSI}^{+}\right]_{\mathrm{WS}}$ bearing deletion of the chromosomal PUB1 demonstrated that pub1- $\triangle$ caused an increase in the amount of Sup35 polymers for $\left[\mathrm{PSI}^{+}\right]_{W S 2}$, albeit to a lesser extent than for the strain with $\left[\mathrm{PSI}^{+}\right]_{\mathrm{S} 7}$, but had no statistically significant effect on the level of Sup35 polymers in the strain with $\left[\mathrm{PSI}^{+}\right]_{W 2}$ (Figure 2).
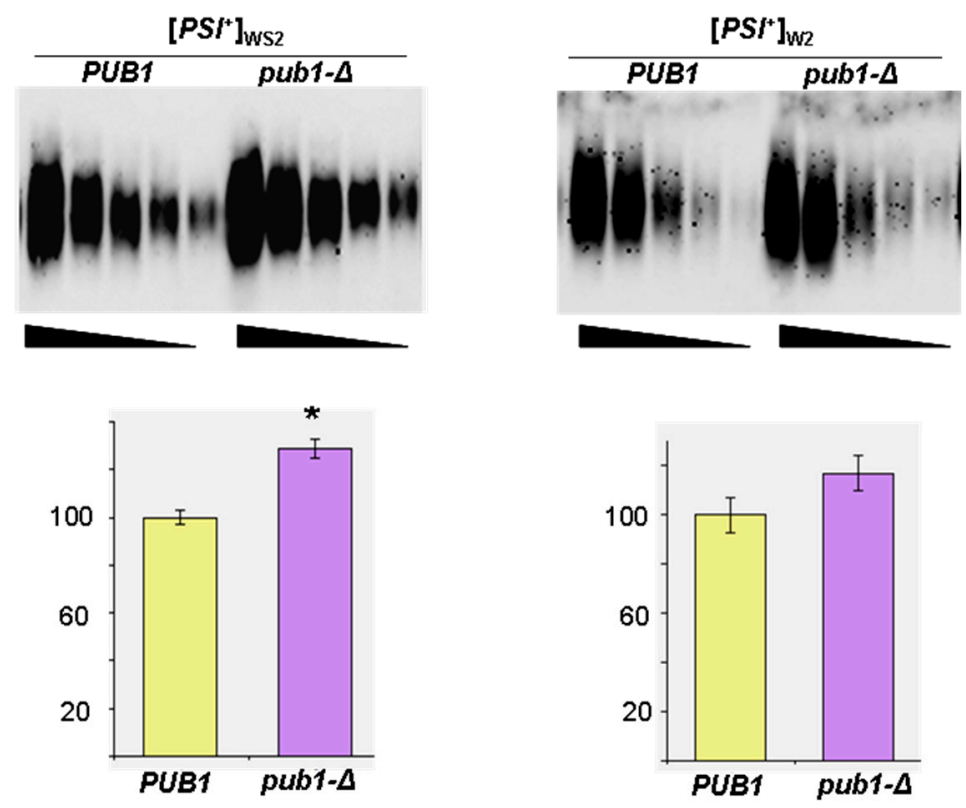

Figure 2. Deletion of PUB1 slightly increases the levels of Sup35 polymers in cells with weak $\left[P S I^{+}\right]$. SDD-AGE analysis of polymerized Sup35: The strains were grown in liquid YPD medium; blots were probed with the polyclonal antibody against Sup35NM; equal amounts of total protein from compared cell lysates were serially diluted in twofold decrements; undiluted samples contained $\sim 180 \mu \mathrm{g}$ of total protein per lane. Four clones of $\left[\mathrm{PSI}^{+}\right]_{\mathrm{WS} 2}$ and $\left[\mathrm{PSI}^{+}\right]_{\mathrm{W} 2}$ derivatives of the 74-D694 strain with deleted (pub1- $\triangle$ ) or without PUB1 deletion (PUB1) grown in liquid YPD were studied, and the abundance of Sup35 polymers in pub1- $\triangle$ and PUB1 strains was calculated as described in the legend to Figure 1 and shown as the percent of the level in the strain bearing wild-type PUB1 \pm SEM in the histograms. A statistically significant increase in the amount of Sup35 polymers caused by $p u b 1-\Delta$ (determined by Student's $t$-test) and indicated by an asterisk $\left({ }^{*}\right)$ was observed for the strain with $\left[\mathrm{PSI}^{+}\right]_{\mathrm{WS} 2}(p<0.05)$, but not with $\left[\mathrm{PSI}^{+}\right]_{\mathrm{W} 2}(p>0.3)$. Typical blot images are presented.

One can suggest that if the lack of Pub1 stimulates Sup35 prion polymerization, then overproduction of this protein should inhibit it. However, quantitative examination of Sup35 prion polymers in the 74-D694 $\left[\mathrm{PSI}^{+}\right]_{\mathrm{S} 7}$ strain overproducing Pub1 demonstrated that excess of this protein did not decrease the level of Sup35 polymers (Figure S3). Notably that the effect of pub1- $\triangle$ is specific for $\left[P^{+} I^{+}\right]$, since this deletion did not affect polymerization of the Rnq1 protein, which is the protein determinant of the $\left[\mathrm{PIN}^{+}\right]$prion $[29,30]$ (Figure S4).

\subsection{The Lack of Upf1, But Not of Pub1, Upf2 or Upf3 Increases Sequestration of Sup45 into Sup35 Prion Polymers}

It was demonstrated earlier that in $\left[\mathrm{PSI}^{+}\right]$cells Sup45 is found mostly in the aggregated state, possibly due to its recruitment by Sup35 prion polymers [24,25], though other studies have not confirmed $\left[\mathrm{PSI}^{+}\right]$-dependent co-aggregation of Sup35 and Sup45 [6,31]. If the Sup35 prion polymers sequester Sup45, then the elevation of their level should further increase aggregation of Sup45. However, sedimentation analysis of lysates of the $\left[\mathrm{PSI}^{+}\right]_{S 7}$ cells with wild-type and deleted PUB1 did 
not show a statistically significant difference in the amount of aggregated Sup45 (Figure 3A). Therefore, the 2-fold increase in the level of Sup35 polymers caused by pub1- $\Delta$ in the cells grown in YPD [23] was not sufficient to secure a noticeable difference of co-aggregated Sup45 in the strains with wild-type and deleted PUB1 grown in the same medium.

(a)

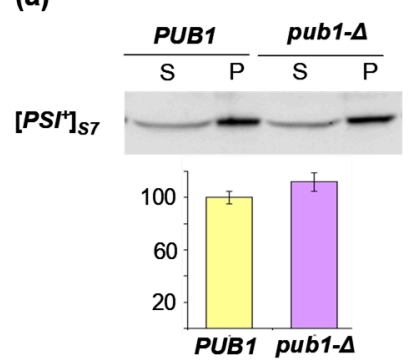

(c)

$\left[P S{ }^{\prime}\right]_{S 7}$

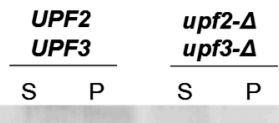

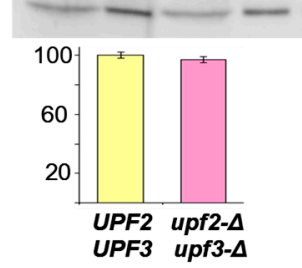

(b)
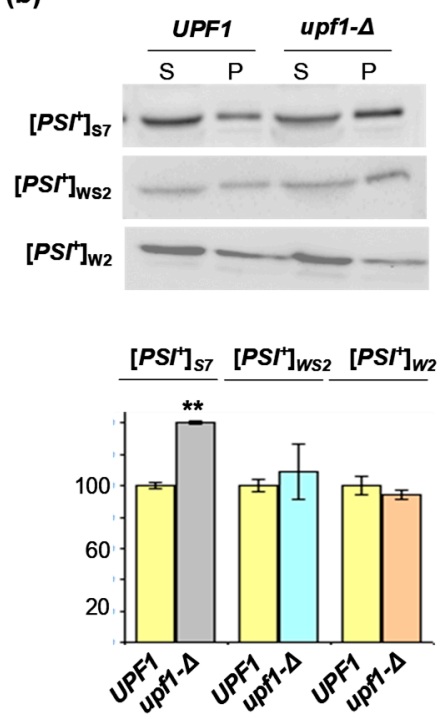

Figure 3. Deletion of UPF1 but not of PUB1, UPF2 or UPF3 increases Sup45 aggregation in cells with $\left[\mathrm{PSI}^{+}\right]_{\mathrm{S} 7}$. Cell lysates were fractionated by ultracentrifugation as described in Materials and Methods. Samples were loaded onto the gel in a volume corresponding to equal amounts of starting lysates, which contained $\sim 200 \mu \mathrm{g}$ of soluble protein. Blots were probed with polyclonal antibody against Sup 45 and levels of Sup 45 in fractions were determined by densitometric analysis of blots. Four clones of each strain were analyzed. S, soluble fraction; P, pellet. The relative abundances of Sup45 in these fractions estimated by densitometry of blots, were calculated as ratios of its signal intensity in the pellet fraction versus the sum of signal intensities in the pellet and soluble fractions and is shown on the histograms as the percent of the level in the strain wild-type for analyzed gene. Typical blot images are presented. The statistical significance of differences in the amount of aggregated Sup 45 in compared strains was estimated by Student's $t$-test. (a) Deletion of PUB1 (pub1- $\Delta$ ) does not cause a statistically significant increase in the amount of aggregated Sup45 $(p>0.08)$, (b) deletion of UPF1 (upf1- $\Delta$ ) causes a statistically significant $(p<0.001)$ increase of the amount of aggregated Sup45 in cells with $\left[\mathrm{PSI}^{+}\right]_{S 7}$, indicated by two asterisks $(* *)$, but not in cells with either $\left[P S I^{+}\right]_{\mathrm{WS} 2}(p>0.2)$ or $\left[P S I^{+}\right]_{\mathrm{W} 2}(p>0.7)$, (c) simultaneous deletion of UPF2 (upf2- $\triangle$ ) and UPF3 (upf3- $\triangle$ ) in $\left[P S I^{+}\right]_{S 7}$ cells does not influence Sup45 aggregation $(p>0.6)$.

\section{Discussion}

In this work we demonstrate the phenomenon of a triple synthetic lethal interaction in yeast; namely, that the combination of the PUB1 and UPF1 deletions with the $\left[P S I^{+}\right]$prion is lethal, albeit it was observed only for the strain bearing the $\left[\mathrm{PSI}^{+}\right]_{S 7}$ variant, manifesting a strong suppression phenotype. We also show that the reason for this lethality is inactivation of Sup45 due to depletion of its soluble and functionally active form caused by sequestration of this protein into Sup35 prion polymers. However, in contrast to upf1- $\triangle$, the deletion of PUB1 did not cause a noticeable increase of Sup45 aggregation, though, depending on growth conditions, its absence caused up to a 2-fold increase in the amount of Sup35 polymers [23]. Unlike PUB1, the UPF1 gene did not affect Sup35 prion polymerization; however, it was involved in maintaining a normal level of soluble Sup45 in cells with strong $\left[\mathrm{PSI}^{+}\right]$, since its deletion in these cells resulted in an approximately 1.5-fold increase of aggregated Sup45. Importantly, though the increase in the level of Sup35 polymers caused by 
pub1- $\Delta$ on its own did not cause observable changes in the aggregation of Sup 45 , it did increase the aggregation of this protein when $p u b 1-\Delta$ was combined with upf1- $\Delta$. Indeed, in this strain a 2-fold increase in the amount of Sup35 polymers and a 1.5-fold increase in Sup 45 co-aggregation resulted in a 3-fold increase in the aggregation of Sup45 as compared to the strain with wild-type PUB1 and UPF1. Remarkably, it was shown earlier that $\left[\mathrm{PSI}^{+}\right]$can inactivate the Sup45 translation termination factor, since deleting one copy of the SUP45 gene in a $\left[\mathrm{PSI}^{+}\right]$but not in a $\left[p s i^{-}\right]$diploid strain caused a noticeable inhibition of cell growth and blocked sporulation [15]. Thus, since a 2-fold decrease of Sup45 amounts is harmful to $\left[\mathrm{PSI}^{+}\right]$cells, it is not surprising that a further 3-fold depletion of soluble Sup45 can be lethal.

Two mechanisms can explain the ability of Pub1 to restrict Sup35 polymerization: (i) Pub1 binds to the ends of Sup35 polymers to restrain their further elongation; and (ii) Pub1 forms complexes with monomeric Sup35, thus inhibiting its ability to join to the ends of a growing polymer. The latter possibility is supported by the observation that Pub1 $\Delta \mathrm{C}$, lacking the short Q-rich C-terminal region, which is critical for its co-polymerization with Sup35 and contains the major site for interaction with monomeric Sup35, suppresses Sup35 polymerization, though less efficiently than full-length Pub1. Most probably, this ability can be attributed to the Pub1 internal low-affinity site for interaction with monomeric Sup35 [23]. Besides, the observation that the lack of the Pub1 protein, which does not interact with monomeric Rnq1 but copolymerizes with it in $\left[\mathrm{PIN}^{+}\right]$cells [32], does not influence Rnq1 polymerization, is also in line with this suggestion. Importantly, this mechanism explains $\left[\mathrm{PSI}^{+}\right]$ variant-dependent effects of $p u b 1-\Delta$ on Sup35 polymerization efficiency. It is known that cells with strong $\left[\mathrm{PSI}^{+}\right]$contain much less soluble Sup35 than cells with weak variants of this determinant $[9,33]$, and therefore, Pub1 can bind a greater proportion of soluble Sup35 in cells with strong $\left[\mathrm{PSI}^{+}\right]$than in cells with weak $\left[\mathrm{PSI}^{+}\right]$. If this is correct, the lack of Pub1 should ensure the most profound effect on Sup35 polymerization in cells with strong $\left[\mathrm{PSI}^{+}\right]$variants. Furthermore, according to the same considerations, excess Pub1 does not decrease the levels of Sup35 polymers in strong $\left[\mathrm{PSI}^{+}\right]$due to insufficient amount of Sup35 monomers available for interaction with Pub1.

Interestingly, it was shown recently that most $\left[\mathrm{PSI}^{+}\right]$variants, which appeared in the absence of Upf proteins, can be eliminated by restoration of the normal levels of these proteins. To explain this effect, it was proposed that inhibition of $\left[\mathrm{PSI}^{+}\right]$prion propagation by Upf proteins may be due to their interaction with soluble Sup35, which distracts this protein from polymerization or, alternatively, with polymerized Sup35, which blocks adding Sup35 monomers to the ends of growing polymers [34]. However, here we show that in contrast to pub1- $\triangle$, deletion of any of the UPF genes does not increase the amount or the size of Sup35 polymers, indicating that at least in cells with $\left[\mathrm{PSI}^{+}\right]$generated in the presence of wild-type UPF genes, Upf proteins are not involved in the process of Sup35 polymerization.

Though, unlike Pub1, Upf1 did not influence Sup35 polymerization, it controlled the level of soluble Sup 45 by inhibiting binding of Sup 45 to Sup 35 polymers, which could be due to its interaction with these polymers. Importantly, the ability of Upf1 to interact with Sup35 polymers was supported by observation of $\left[\mathrm{PSI}^{+}\right]$-dependent co-sedimentation of these proteins [24], as well as co-localization of their fusions with alternative fluorescent proteins [34]. Nevertheless, the effect of Upf1 on interaction of Sup45 with Sup35 polymers seems surprising, since it is known that only Upf2 and Upf3, but not Upf1 compete with Sup45 for binding to monomeric Sup35, which agrees with a spatial separation of corresponding binding sites in Sup35. Indeed, it was shown that Upf1 interacts with Sup35 through a proximal site in its C-terminal domain, while Upf2, Upf3 and Sup45 bind to the overlapping sites located in a distal region of this Sup35 domain [27]. Thus, it remains to suggest that Upf proteins interact differently with monomeric and polymeric Sup35. It is probable that the site for Upf1 binding in Sup35 involved in a polymer is exposed, while the site for Upf2, Upf3 and Sup45 is not, and the lack of Upf1 makes this Upf2/Upf3/Sup45-specific site available for interaction with Sup45 (Figure 4). This also explains the inability of UPF2 and UPF3 deletions to influence binding of Sup45 to polymerized Sup35. 
Proteins whose absence affects $\left[\mathrm{PSI}^{+}\right]$formation, propagation and/or phenotypic manifestation can be divided into two classes. The first class involves cytosolic chaperones of the Hsp40, Hsp70 and Hsp100 families as well as the chaperone sorting factor Cur1 $[19,35,36]$. The second class includes functionally unrelated non-chaperone proteins, such as vacuolar proteases $\operatorname{Pr} A$ and $\operatorname{PrB}$ [22], Upf1/2/3 proteins controlling NMD and nonsense codon readthrough [34], as well as Siw14 and Arg82, enzymes involved in the inositol polyphosphate biosynthetic pathway [21]. Mechanisms of action of these proteins remain elusive, with the exception of $\operatorname{PrA}$ and $\operatorname{PrB}$ proteases, which cleave off an important part of the Sup35 prion-forming domain.
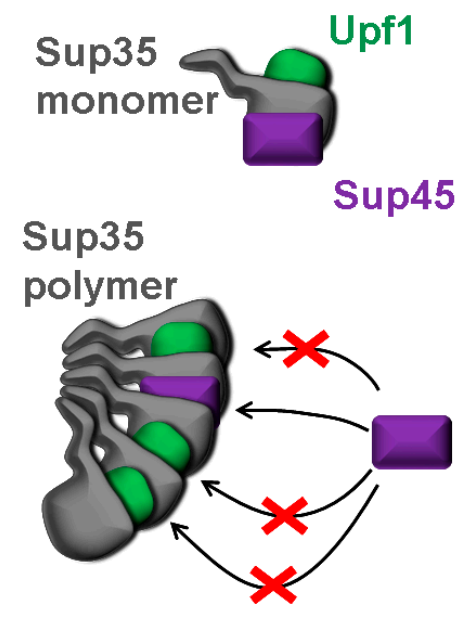

Figure 4. Schematic representation of the suggested mechanism mediating the effect of Upf1 on binding of Sup45 to polymeric Sup35. Upf1 and Sup45 interact with monomeric Sup35 independently. Sup45 binds to Sup35 involved in a polymer only if Sup35 is not bound to Upf1.

Notably, besides the anti-prion systems counteracting $\left[\mathrm{PSI}^{+}\right]$formation, yeast cells contain systems preventing $\left[\mathrm{PSI}^{+}\right]$cytotoxicity, which are also based on both chaperone and non-chaperone proteins. One of these systems is based on the nascent polypeptide-associated complex representing a highly conserved triad of proteins that bind near the ribosome exit tunnel. It was shown that deletion of subunits of this complex rescues toxicity associated with the strong $\left[\mathrm{PSI}^{+}\right]$prion, which can be explained by changes in chaperone balance and distribution, whereby the folding of the prion protein is improved and the prion is rendered nontoxic [37]. Another chaperone-assisted [PSI $\left.{ }^{+}\right]$detoxification system is based on the Hsp40 Sis1 chaperone [38]. The mechanism of the toxicity, which is rescued by Sis1, is not yet clear, but most probably it is not related to Sup45 depletion. Other $\left[\mathrm{PSI}^{+}\right]$anti-toxic systems described involve non-chaperone proteins. One of them, revealed here, consists of two proteins, Pub1 and Upf1; the former saves the cell from excessive Sup35 polymerization, while the latter alleviates binding of Sup45 to Sup35 polymers. One more such system involves the actin assembly protein Sla2, whose protective effect is unlikely to involve sequestration of Sup45 into prion aggregates [13]. Thus, it is possible that at least two proteins, Sis1 and Sla2, alleviate $\left[\mathrm{PSI}^{+}\right]$toxicity by preventing sequestration of essential cellular components other than Sup45 into prion aggregates. This suggests that different $\left[\mathrm{PSI}^{+}\right]$detoxification systems may protect the cell from the defects of various essential processes not related to translation. Indeed, Sup35 was shown to possess essential functions unrelated to its role in the translation termination $[39,40]$, which can be compromised by its prion aggregation.

Finally, it should be stressed that although the role of $\left[\mathrm{PSI}^{+}\right]$in yeast biology is still unclear, it is possible that even if most commonly appearing $\left[\mathrm{PSI}^{+}\right]$variants are harmful, some of them can be beneficial and due to this, yeast has developed special systems for self-protection from the deleterious side effects of this prion. 


\section{Materials and Methods}

\subsection{Yeast Strains and Growth Conditions}

All experiments described in this study were performed with the use of the $\left[\mathrm{psi}^{-}\right]\left[\mathrm{pin}^{-}\right]$derivative of the S. cerevisiae strain 74-D694 (MATa ura3-52 leu2-3,112 trp1-289 his3- 200 ade1-14), as well as its variants carrying $\left[\mathrm{PIN}^{+}\right]$and either strong $\left[\mathrm{PSI}^{+}\right]$, originally present in this strain and designated here as $\left[P S I^{+}\right]_{S 7}[41]$ or weak $\left[P S I^{+}\right]_{W S 2}$ and $\left[P S I^{+}\right]_{W 2}$ which were generated in the $\left[p i^{-}\right]\left[P I N^{+}\right]$ background by transient overproduction of Sup35 and selected by the ability to suppress the ade1-14 $\mathrm{UGA}$ mutation [42]. The construction of genetically-modified variants of this strain is described in the next section. Yeast were grown at $30{ }^{\circ} \mathrm{C}$ in rich (YPD, $1 \%$ yeast extract, $2 \%$ peptone, $2 \%$ glucose) or synthetic (SC, $0.67 \%$ yeast nitrogen base, $2 \%$ glucose supplemented with appropriate amounts of the required amino acids or bases) media. All growth assays were made in triplicate.

\subsection{Plasmids and Nucleic Acid Manipulation}

Plasmids used in this study are presented in Table 3. To generate the plasmids pRS316-PUB1 and YEplac181-PUB1, the PUB1 gene harboring the EcoRI-XbaI fragment of YEplac195-PUB1 was inserted into the same sites of the pRS316 and YEplac181 plasmids, respectively. The EcoRI-XbaI fragment of YEplac195-PUB1 $\Delta C$ was inserted into the same site of pRS316 to generate the pRS316-PUB1 $\triangle \mathrm{C}$ plasmid. To construct the pRS315-UPF1 and YEplac181-UPF1 plasmids, the UPF1 gene harboring the PstI-PvuII fragment of YEplac112-UPF1 was inserted into the PstI and SmaI sites of the pRS315 and YEplac181 plasmids, respectively. The UPF1 gene was disrupted in the 74-D694 $\left[P I N^{+}\right]\left[P S I^{+}\right]_{S 7}$ strain using the upf1::URA3 disruption cassette, as described in Reference [43]. The upf2::URA3 gene disruption cassette was obtained by PCR amplification using the primers 5'-GTGTACTGGAACGGTCCAATA-3' and 5'-ATACACTGGCAGTTTGCTCCA-3' and the genomic DNA of the Y41 strain (MATa his4-38 SUF1-1 ura3-52 leu2-3 trp1-1 UPF2::URA3), which is the UPF2 disruption derivative of the PLY18 strain [43]. This cassette was used to disrupt UPF2 in the 74-D694 $\left[\mathrm{PIN}^{+}\right]\left[P S I^{+}\right]_{S 7}$ strain. Similarly, the upf3::kanMX gene disruption cassette, obtained by PCR amplification using primers $5^{\prime}$-CCCCATGTAAATCATCCAAT- $3^{\prime}$ and $5^{\prime}$-TGGAGTCATCTTTCTTCATG-3', and the genomic DNA of the upf3- $\Delta$ derivative of the BY4742 strain (MAT $\alpha$ his3- $\Delta 1$ leu2- $\Delta 0$ lys2- $\Delta 0$ ura3- $\triangle 0$ upf3::kanMX) obtained from EUROSCARF, was used to select the G418-resistant UPF3 disruptant of the 74-D694 $\left[\mathrm{PIN}^{+}\right]\left[\mathrm{PSI}^{+}\right]_{\mathrm{S} 7}$ strain. The PUB1 disruptant of the 74-D694 $\left[P_{I N}^{+}\right]\left[P S I^{+}\right]_{S 7}$ strain and the procedures for the PUB1 gene disruption in 74-D694 derivatives with $\left[P S I^{+}\right]_{\mathrm{WS} 2}$ and $\left[P S I^{+}\right]_{\mathrm{W} 2}$ using the $p u b 1:: T R P 1$ disruption cassette were the same as described earlier [23]. Disruption of the above-mentioned genes was verified by PCR analysis.

\subsection{Electrophoresis and Blotting}

SDS-PAGE was performed according to the standard protocol in $10 \%$ polyacrylamide gels and SDD-AGE (Semi denaturing detergent-agarose gel electrophoresis) as described previously [7,44]. Protein loads were equalized for each gel. For the SDD-AGE analysis of amyloid polymers we used horizontal $1.8 \%$ agarose gels in the Tris-Acetate-EDTA (TAE) buffer with $0.1 \%$ SDS. Lysates were incubated in sample buffer $(0.5 \times$ TAE, $2 \%$ SDS, $5 \%$ glycerol and $0.05 \%$ Bromophenol Blue) for $5 \mathrm{~min}$ at room temperature. After electrophoresis, proteins were transferred from gels to nitrocellulose membrane sheets (Thermo Scientific, Waltham, MA, USA) by vacuum-assisted capillary blotting for $8 \mathrm{~h}$ (agarose gels), or electrophoretically (polyacrylamide gels). Bound antibody was detected using the enhanced chemiluminescence (ECL) West Dura system (Thermo Scientific, Waltham MA, USA). It should be noted that detergents (SDS or sarcosyl) in non-boiled samples increase degradation of Sup35 monomers. This can result in the absence of Sup35 monomer bands in SDD-AGE gels. Rabbit polyclonal antibodies against yeast Sup35NM (Sup35 lacking the C-terminal domain responsible for translation termination activity), Sup45 [39,45] and Pub1 [32] were used. Densitometry measurements were performed using ImageJ software. 
Table 3. Plasmids.

\begin{tabular}{|c|c|c|}
\hline Plasmids & Characteristics & Source \\
\hline YEplac181 & Multicopy LEU2 plasmid & [46] \\
\hline YEplac181-PUB1 & Multicopy LEU2 plasmid harboring the PUB1 gene & This work \\
\hline YEplac181-SUP35C & Multicopy LEU2 plasmid encoding Sup35C & [47] \\
\hline Yeplac181-UPF1 & Multicopy LEU2 plasmid harboring the UPF1 gene & This work \\
\hline YEplac195 & Multicopy URA3 plasmid & [46] \\
\hline YEplac195-PUB1 & Multicopy URA3 plasmid harboring the PUB1 gene & [32] \\
\hline YEplac195-PUB1 $\Delta C$ & Multicopy URA3 plasmid encoding Pub1 $\triangle \mathrm{C}$ & [23] \\
\hline YEplac195-SUP45 & Multicopy URA3 plasmid harboring the SUP45 gene & [48] \\
\hline YEplac112-UPF1 & Multicopy TRP1 plasmid harboring the UPF1 gene & [49] \\
\hline pRS315 & Centromeric $L E U 2$ plasmid & [50] \\
\hline pRS315-SUP35C & Centromeric LEU2 plasmid encoding Sup35C & [40] \\
\hline pRS315-SUP45 & Centromeric LEU2 plasmid harboring the SUP45 gene & [45] \\
\hline pRS315-UPF1 & Centromeric LEU2 plasmid harboring the UPF1 gene & This work \\
\hline pRS316 & Centromeric URA3 plasmid & [50] \\
\hline pRS316- PUB1 & Centromeric URA3 plasmid harboring the PUB1 gene & This work \\
\hline pRS316- PUB1 $\Delta C$ & Centromeric $U R A 3$ plasmid encoding Pub1 $\triangle \mathrm{C}$ & This work \\
\hline pEMBLyex4 $\left(\Delta\right.$ LEU2 $\left._{\mathrm{d}}\right)-3 \mathrm{ATG}$ & Multicopy URA3 plasmid encoding Sup35C & [51] \\
\hline PPUB1::TRP1 & Plasmid encoding pub1::TRP1 disruption cassette & [23] \\
\hline $\mathrm{pKOM}$ & Plasmid encoding upf1::URA3 disruption cassette & [43] \\
\hline
\end{tabular}

\subsection{Preparation and Fractionation of Yeast Cell Lysates}

Yeast cells grown in liquid selective media to $\mathrm{OD}_{600}$ of 2.5 were harvested, washed in water and disrupted by beating with glass beads (Bullet Blender, Next Advance, Troy, NY, USA) in buffer A: $30 \mathrm{mM}$ Tris- $\mathrm{HCl}, \mathrm{pH} 7.4,150 \mathrm{mM} \mathrm{NaCl}, 1 \mathrm{mM}$ dithiothreitol with $10 \mathrm{mM}$ phenylmethylsulfonyl fluoride and CompleteTM protease inhibitor cocktail (Roche Applied Science, Indianapolis, IN, USA) to prevent proteolytic degradation. After centrifugation of crude lysates at $1500 \times g$ for $4 \mathrm{~min}$, cell debris containing glass beads was washed in buffer A, containing $1 \%$ Triton X-100 or $1 \%$ SDS, if polymers of Sup35 and Rnq1 were analyzed by SDD-AGE. To analyze the content of soluble and aggregated Sup45 by centrifugation, cells were grown to $\mathrm{OD}_{600}$ of 2.0. The lysates were prepared in buffer $\mathrm{A}$, crowding agent, Ficoll PM400 at a concentration close to the physiological concentration of macromolecules ( $200 \mathrm{mg} / \mathrm{mL}$ ) and $20 \mathrm{mM}$ EDTA dissociating ribosomes to subunits. Lysates $(0.05 \mathrm{~mL})$ were underlaid with the same volume of $30 \%$ sucrose pads made in buffer A and centrifuged at $100,000 \times g, 4{ }^{\circ} \mathrm{C}$ for $90 \mathrm{~min}$. Pellets were resuspended in volumes equal to the volumes of the ultracentrifuged lysates. The resulting supernatant and pellet fractions were analyzed by Western blotting using antibodies against Sup45.

\subsection{Determination of the Efficiency of Nonsense Codon Readthrough}

To measure the efficiency of nonsense codon readthrough, plasmids of a pDB series carrying tandem Renilla and firefly luciferase genes separated by a single in-frame UGA(C) codon or a corresponding sense codon control were used [28,52]. Assays were performed with a dual luciferase reporter assay system (Promega, Madison, WI, USA), as described [53] with minimal modifications using a Glomax 20/20 luminometer (Promega, Madison, WI, USA). All assays were repeated three times. The readthrough in each strain is expressed as the ratio of firefly luciferase activity/Renilla luciferase activity (nonsense codon between luciferase genes) divided by the ratio of firefly luciferase activity/Renilla luciferase activity (sense codon between luciferase genes). For further details, see Reference [54].

Supplementary Materials: Supplementary Materials can be found at http:/ / www.mdpi.com/1422-0067/19/11/ $3663 / \mathrm{s} 1$.

Author Contributions: Conceptualization, M.D.T.-A.; formal analysis, V.N.U., O.V.M., A.A.D. and M.D.T.-A.; investigation, V.N.U., O.V.M. and A.A.D.; writing-original draft preparation, M.D.T.-A.; writing一review and editing, O.V.M. and M.D.T.-A.; supervision, M.D.T.-A. 
Funding: The research was partially funded by a grant from the Russian Foundation for Basic Research \# 17-04-00032 (V.N.U., O.V.M. and M.D.T.-A.). The study of [PSI+]-dependent co-aggregation of Sup35 and Sup45 was supported by a grant from the Russian Science Foundation \# 17-14-01092 (A.A.D. and M.D.T.-A.).

Acknowledgments: The authors are grateful to V. Kushnirov for critical reading of the manuscript and to M. Agaphonov for drawing the scheme of the interaction of Sup35 with Upf1 and Sup45.

Conflicts of Interest: The authors declare no conflicts of interest.

\section{References}

1. Chiti, F.; Dobson, C.M. Protein misfolding, functional amyloid and human disease. Annu. Rev. Biochem. 2006, 75, 333-366. [CrossRef] [PubMed]

2. Nizhnikov, A.A.; Antonets, K.S.; Inge-Vechtomov, S.G. Amyloids: From pathogenesis to function. Biochemistry 2015, 80, 1127-1144. [CrossRef] [PubMed]

3. Shkundina, I.S.; Ter-Avanesyan, M.D. Prions. Biochemistry 2007, 72, 1519-1536. [CrossRef] [PubMed]

4. Wickner, R.B. Yeast and fungal prions. Cold Spring Harb. Perspect. Biol. 2016, 8, a023531. [CrossRef] [PubMed]

5. Paushkin, S.V.; Kushnirov, V.V.; Smirnov, V.N.; Ter-Avanesyan, M.D. Propagation of the yeast prion-like [psi+] determinant is mediated by oligomerization of the SUP35-encoded polypeptide chain release factor. EMBO J. 1996, 15, 3127-3134. [CrossRef] [PubMed]

6. Patino, M.M.; Liu, J.J.; Glover, J.R.; Lindquist, S. Support for the prion hypothesis for inheritance of a phenotypic trait in yeast. Science 1996, 273, 622-626. [CrossRef] [PubMed]

7. Kryndushkin, D.S.; Alexandrov, I.M.; Ter-Avanesyan, M.D.; Kushnirov, V.V. Yeast $\left[P S I^{+}\right]$prion aggregates are formed by small Sup35 polymers fragmented by Hsp104. J. Biol. Chem. 2003, 278, 49636-49643. [CrossRef] [PubMed]

8. Derkatch, I.L.; Chernoff, Y.O.; Kushnirov, V.V.; Inge-Vechtomov, S.G.; Liebman, S.W. Genesis and variability of $\left[\mathrm{PSI}^{+}\right]$prion factors in Saccharomyces cerevisiae. Genetics 1996, 144, 1375-1386. [PubMed]

9. Kochneva-Pervukhova, N.V.; Chechenova, M.B.; Valouev, I.A.; Kushnirov, V.V.; Smirnov, V.N.; Ter-Avanesyan, M.D. $\left[\mathrm{PSI}^{+}\right]$prion generation in yeast: Characterization of the 'strain' difference. Yeast 2001, 18, 489-497. [CrossRef] [PubMed]

10. Tanaka, M.; Chien, P.; Naber, N.; Cooke, R.; Weissman, J.S. Conformational variations in an infectious protein determine prion strain differences. Nature 2004, 428, 323-328. [CrossRef] [PubMed]

11. Krishnan, R.; Lindquist, S.L. Structural insights into a yeast prion illuminate nucleation and strain diversity. Nature 2005, 435, 765-772. [CrossRef] [PubMed]

12. Reidy, M.; Masison, D.C. Modulation and elimination of yeast prions by protein chaperones and co-chaperones. Prion 2011, 5, 245-249. [CrossRef] [PubMed]

13. Ganusova, E.E.; Ozolins, L.N.; Bhagat, S.; Newnam, G.P.; Wegrzyn, R.D.; Sherman, M.Y.; Chernoff, Y.O. Modulation of prion formation, aggregation, and toxicity by the actin cytoskeleton in yeast. Mol. Cell. Biol. 2006, 26, 617-629. [CrossRef] [PubMed]

14. Derkatch, I.L.; Bradley, M.E.; Liebman, S.W. Overexpression of the SUP45 gene encoding a Sup35p-binding protein inhibits the induction of the de novo appearance of the $\left[\mathrm{PSI}^{+}\right]$prion. Proc. Natl. Acad. Sci. USA 1998, 95, 2400-2405. [CrossRef] [PubMed]

15. Dagkesamanskaya, A.R.; Ter-Avanesyan, M.D. Interaction of the yeast omnipotent suppressors SUP1(SUP45) and SUP2(SUP35) with non-Mendelian factors. Genetics 1991, 128, 513-520. [PubMed]

16. Kiktev, D.; Inge-Vechtomov, S.; Zhouravleva, G. Prion-dependent lethality of sup45 mutants in Saccharomyces cerevisiae. Prion 2007, 1, 136-143. [CrossRef] [PubMed]

17. True, H.L.; Lindquist, S.L. A yeast prion provides a mechanism for genetic variation and phenotypic diversity. Nature 2000, 407, 477-483. [CrossRef] [PubMed]

18. Tyedmers, J.; Madariaga, M.L.; Lindquist, S. Prion switching in response to environmental stress. PLoS Biol. 2008, 6, e294. [CrossRef] [PubMed]

19. Wickner, R.B.; Shewmaker, F.P.; Bateman, D.A.; Edskes, H.K.; Gorkovskiy, A.; Dayani, Y.; Bezsonov, E.E. Yeast prions: Structure, biology, and prion-handling systems. Microbiol. Mol. Biol. Rev. 2015, 79, 1-17. [CrossRef] [PubMed]

20. Mcglinchey, R.P.; Kryndushkin, D.; Wickner, R.B. Suicidal [PSI+] is a lethal yeast prion. Proc. Natl. Acad. Sci. USA 2011, 108, 5337-5341. [CrossRef] [PubMed] 
21. Wickner, R.B.; Bezsonov, E.E.; Son, M.; Ducatez, M.; Dewilde, M.; Edskes, H.K. Anti-prion systems in yeast and inositol polyphosphates. Biochemistry 2018, 57, 1285-1292. [CrossRef] [PubMed]

22. Okamoto, A.; Hosoda, N.; Tanaka, A.; Newnam, G.P.; Chernoff, Y.O.; Hoshino, S.I. Proteolysis suppresses spontaneous prion generation in yeast. J. Biol. Chem. 2017, 292, 20113-20124. [CrossRef] [PubMed]

23. Urakov, V.N.; Mitkevich, O.V.; Safenkova, I.V.; Ter-Avanesyan, M.D. Ribosome-bound Pub1 modulates stop codon decoding during translation termination in yeast. FEBS J. 2017, 284, 1914-1930. [CrossRef] [PubMed]

24. Czaplinski, K.; Ruiz-Echevarria, M.J.; Paushkin, S.V.; Han, X.; Weng, Y.; Perlick, H.A.; Dietz, H.C.; Ter-Avanesyan, M.D.; Peltz, S.W. The surveillance complex interacts with the translation release factors to enhance termination and degrade aberrant mRNAs. Genes Dev. 1998, 12, 1665-1677. [CrossRef] [PubMed]

25. Paushkin, S.V.; Kushnirov, V.V.; Smirnov, V.N.; Ter-Avanesyan, M.D. Interaction between yeast Sup45p (eRF1) and Sup35p (eRF3) polypeptide chain release factors: Implications for prion-dependent regulation. Mol. Cell. Biol. 1997, 17, 2798-2805. [CrossRef] [PubMed]

26. He, F.; Broun, A.H.; Jacobson, A. Upf1p, Nmd2p, and Upf3p are interacting components of the yeast nonsense-mediated mRNA decay pathway. Mol. Cell. Biol. 1997, 17, 1580-1594. [CrossRef] [PubMed]

27. Wang, W.; Czaplinski, K.; Rao, Y.; Peltz, S.W. The role of Upf proteins in modulating the translation readthrough of nonsense-containing transcripts. EMBO J. 2001, 20, 880-890. [CrossRef] [PubMed]

28. Salas-Marco, J.; Bedwell, D.M. GTP hydrolysis by eRF3 facilitates stop codon decoding during eukaryotic translation termination. Mol. Cell. Biol. 2004, 24, 7769-7778. [CrossRef] [PubMed]

29. Derkatch, I.L.; Bradley, M.E.; Hong, J.Y.; Liebman, S.W. Prions affect the appearance of other prions: The story of [PIN ${ }^{+}$. Cell 2001, 106, 171-182. [CrossRef]

30. Sondheimer, N.; Lindquist, S. Rnq1: An epigenetic modifier of protein function in yeast. Mol. Cell 2000, 5, 163-172. [CrossRef]

31. Eaglestone, S.S.; Cox, B.S.; Tuite, M.F. Translation termination efficiency can be regulated in Saccharomyces cerevisiae by environmental stress through a prion-mediated mechanism. EMBO J. 1999, 18, 1974-1981. [CrossRef] [PubMed]

32. Urakov, V.N.; Vishnevskaya, A.B.; Alexandrov, I.M.; Kushnirov, V.V.; Smirnov, V.N.; Ter-Avanesyan, M.D. Interdependence of amyloid formation in yeast: Implications for polyglutamine disorders and biological functions. Prion 2010, 4, 45-52. [CrossRef] [PubMed]

33. Zhou, P.; Derkatch, I.L.; Uptain, S.M.; Patino, M.M.; Lindquist, S.; Liebman, S.W. The yeast non-Mendelian factor $\left[E T A^{+}\right]$is a variant of $\left[\mathrm{PSI}^{+}\right]$, a prion-like form of release factor eRF3. EMBO J. 1999, 18, 1182-1191. [CrossRef] [PubMed]

34. Son, M.; Wickner, R.B. Nonsense-mediated mRNA decay factors cure most $\left[\mathrm{PSI}^{+}\right]$prion variants. Proc. Natl. Acad. Sci. USA 2018, 2, E1184-E1193. [CrossRef] [PubMed]

35. Chernova, T.A.; Wilkinson, K.D.; Chernoff, Y.O. Physiological and environmental control of yeast prions. FEMS Microbiol. Rev. 2014, 38, 326-344. [CrossRef] [PubMed]

36. Barbitoff, Y.A.; Matveenko, A.G.; Moskalenko, S.E.; Zemlyanko, O.M.; Newnam, G.P.; Patel, A.; Chernova, T.A.; Chernoff, Y.O.; Zhouravleva, G.A. To CURe or not to CURe? Differential effects of the chaperone sorting factor Cur1 on yeast prions are mediated by the chaperone Sis1. Mol. Microbiol. 2017, 105, 242-257. [CrossRef] [PubMed]

37. Keefer, K.M.; True, H.L. Prion-associated toxicity is rescued by elimination of cotranslational chaperones. PLoS Genet. 2016, 12, e1006431. [CrossRef] [PubMed]

38. Kirkland, P.A.; Reidy, M.; Masison, D.C. Functions of yeast Hsp40 chaperone Sis1p dispensable for prion propagation but important for prion curing and protection from prion toxicity. Genetics 2011, 188, 565-577. [CrossRef] [PubMed]

39. Valouev, I.A.; Kushnirov, V.V.; Ter-Avanesyan, M.D. Yeast polypeptide chain release factors eRF1 and eRF3 are involved in cytoskeleton organization and cell cycle regulation. Cell Motil. Cytoskelet. 2002, 52, 161-173. [CrossRef] [PubMed]

40. Urakov, V.N.; Valouev, I.A.; Kochneva-Pervukhova, N.V.; Packeiser, A.N.; Vishnevsky, A.Y.; Glebov, O.O.; Smirnov, V.N.; Ter-Avanesyan, M.D. N-terminal region of Saccharomyces cerevisiae eRF3 is essential for the functioning of the eRF1/eRF3 complex beyond translation termination. BMC Mol. Biol. 2006, 7, 34. [CrossRef] [PubMed]

41. Bailleul, P.A.; Newnam, G.P.; Steenbergen, J.N.; Chernoff, Y.O. Genetic study of interactions between the cytoskeletal assembly protein Sla1. Genetics 1999, 153, 81-94. [PubMed] 
42. Dergalev, A.A.; Ivannikov, R.I.; Ter-Avanesyan, M.D.; Kushnirov, V.V. Yeast Sup35 prion structure: Three parts, two types, many variants. PLoS Genet. 2018, in press.

43. Cui, Y.; Hagan, K.W.; Zhang, S.; Peltz, S.W. Identification and characterization of genes that are required for the accelerated degradation of mRNAs containing a premature translational termination codon. Genes Dev. 1995, 9, 423-436. [CrossRef] [PubMed]

44. Kushnirov, V.V.; Alexandrov, I.M.; Mitkevich, O.V.; Shkundina, I.S.; Ter-Avanesyan, M.D. Purification and analysis of prion and amyloid aggregates. Methods 2006, 39, 50-55. [CrossRef] [PubMed]

45. Valouev, I.A.; Urakov, V.N.; Kochneva-Pervukhova, N.V.; Smirnov, V.N.; Ter-Avanesyan, M.D. Translation termination factors function outside of translation: Yeast eRF1 interacts with myosin light chain, Mlc1p, to effect cytokinesis. Mol. Microbiol. 2004, 53, 687-696. [CrossRef] [PubMed]

46. Gietz, R.D.; Sugino, A. New yeast-Escherichia coli shuttle vectors constructed with in vitro mutagenized yeast genes lacking six-base pair restriction sites. Gene 1998, 74, 527-534. [CrossRef]

47. Valouev, I.A.; Fominov, G.V.; Sokolova, E.E.; Smirnov, V.N.; Ter-Avanesyan, M.D. Elongation factor eEF1B modulates functions of the release factors eRF1 and eRF3 and the efficiency of translation termination in yeast. BMC Mol. Biol. 2009, 10, 60. [CrossRef] [PubMed]

48. Urakov, V.N.; Valouev, I.A.; Lewitin, E.I.; Paushkin, S.V.; Kosorukov, V.S.; Kushnirov, V.V.; Smirnov, V.N.; Ter-Avanesyan, M.D. Itt1p, a novel protein inhibiting translation termination in Saccharomyces cerevisiae. BMC Mol. Biol. 2001, 2, 9. [CrossRef]

49. Weng, Y.; Czaplinski, K.; Peltz, S.W. Genetic and biochemical characterization of mutations in the ATPase and helicase regions of the Upf1 protein. Mol. Cell Biol. 1996, 16, 5477-5490. [CrossRef] [PubMed]

50. Sikorski, R.S.; Hieter, P. A system of shuttle vectors and yeast host strains designed for efficient manipulation of DNA in Saccharomyces cerevisiae. Genetics 1989, 122, 19-27. [PubMed]

51. Ter-Avanesyan, M.D.; Kushnirov, V.V.; Dagkesamanskaya, A.R.; Didichenko, S.A.; Chernoff, Y.O.; Inge-Vechtomov, S.G.; Smirnov, V.N. Deletion analysis of the SUP35 gene of the yeast Saccharomyces cerevisiae reveals two non-overlapping functional regions in the encoded protein. Mol. Microbiol. 1993, 7, 683-692. [CrossRef] [PubMed]

52. Keeling, K.M.; Lanier, J.; Du, M.; Salas-Marko, J.; Gao, L.; Kaenjak-Angeletti, A.; Bedwell, D.M. Leaky termination at premature stop codons antagonizes nonsense-mediated mRNA decay in S. cerevisiae. RNA 2004, 10, 691-703. [CrossRef] [PubMed]

53. McNabb, D.S.; Reed, R.; Marciniak, R.A. Dual luciferase assay system for rapid assessment of gene expression in Saccharomyces cerevisiae. Eukaryot. Cell 2005, 4, 1539-1549. [CrossRef] [PubMed]

54. Jacobs, J.L.; Dinman, J.D. Systematic analysis of bicistronic reporter assay data. Nucleic Acids Res. 2004, 32, e160. [CrossRef] [PubMed]

(C) 2018 by the authors. Licensee MDPI, Basel, Switzerland. This article is an open access article distributed under the terms and conditions of the Creative Commons Attribution (CC BY) license (http://creativecommons.org/licenses/by/4.0/). 